\title{
The Design of Blue Emitting Materials Based on Spirosilabifluorene Derivatives
}

\author{
Miao Sun, Ben Niu, and Jingping Zhang \\ Faculty of Chemistry, Northeast Normal University, Changchun 130024, China \\ zhangjp162@nenu.edu.cn
}

\begin{abstract}
Ground state geometries and electronic properties of four experimentally reported spirosilabifluorene derivatives are calculated by $\mathrm{HF}(\mathrm{DFT}) / 6-31 \mathrm{G}^{*}$ method. Their first excited state geometries are investigated using CIS/6-31G* method. The absorption and emission spectra are evaluated by TD-B3LYP/6-31G* and show an excellent agreement with the experimental data. The " $\mathrm{CH} / \mathrm{N}$ substituted spirosilabifluorene derivatives are also investigated. Compared to the pristine molecule, no significant change of the emission wavelengths are found for the " $\mathrm{CH}$ " $/ \mathrm{N}$ substituted derivatives. Furthermore, we find that the performance and the optical properties of these derivatives can be improved through oligomerization or addition of a phenyl group.
\end{abstract}

Keywords: spirosilabifluorene, TD-DFT, HF, emission, oscillator strength.

\section{Introduction}

Organic light-emitting diodes (OLEDs) have been the subjects of intensive investigation, since they were reported by Tang et al. [1] The major challenges remain, including the need to develop a blue electroluminescence emitter material, which is essential for the development of a full color display based on the color changing medium technology or white emission. [2, 3] OLEDs based on silicon derivatives exhibit excellent performance with external electroluminescence quantum efficiencies close to the theoretical limit for a fluorescent material. [4] Compared with spirobifluorene, spirosilabifluorene may have an improved morphological stability in films.[5] They have also been extensively studied as charge transport materials, and the frontier molecular orbitals give the main contribution to charge-transfer interactions. $[6,7]$

Recently, a series of aryl-substituted 9,9'-spiro-9-silabifluorene (SSF) derivatives, 2,2'-di-tert-butyl-7,7'-diphenyl-9,9' -spiro-9-silabifluorene (1a), 2,2'-di-tert-butyl-7,7' -dipyridin-2-yl-9,9'-spiro-9-silabifluorene (1b), 2,2' -di-tert-butyl-7,7' -diphenyl-4-yl9,9'-spiro-9-silabifluorene (1c), and 2,2'-di-tert-butyl-7,7'-bis(2,2'-bipyridin-6-yl)-9, 9'- spiro-9-silabifluorene (1d) were prepared by Lee et al.[4] The absorption spectrum of each of the novel spiro-linked siloles was found showing a significant red shift relative to that of the corresponding carbon analogue. [8] The corresponding solid-state films were reported to exhibit intense violet-blue emission $\left(\lambda_{\mathrm{PL}}=398-415 \mathrm{~nm}\right)$. 
In this contribution, we have investigated the optical and electronic properties of spirosilabifluorene derivatives (1a-1d) with the aim to give a further explanation of the experimental results, and then design new photoluminescence materials based on selected approaches for spirosilabifluorene based materials. "CH"/N substitution approach, which has been cited as an efficient approach for tuning of the emitting color in the case of $\mathrm{Mq3}(\mathrm{M}=\mathrm{Al},[9,10] \mathrm{Ga}$ [9] and $\mathrm{q}=8$-hydroxyquinoline $)$ based fluorescent materials, has not exploited yet in the case of spirosilabifluorene based materials. Thus, "CH"/N substituted spirosilabifluorene derivatives are investigated. With the aim to investigate the ending groups for enhancing the oscillator strength $(f)$, we design the derivatives with and without $t$-Bu and phenyl groups.

\section{Computational Details}

The structures of spirosilabifluorene derivatives (1a-1d) and the designed derivatives were provided in Figure 1. For the sake of comparison, spirosilabifluorene (SSF) was used as parent molecule. Two SSF derivatives with phenyl (1e) and $t$-Bu (1f) groups were chosen to investigate the ending group effect on $f$. The other substituted models used in our calculations, obtained by a systematic substitution of " $\mathrm{CH}$ " by $\mathrm{N}$ atoms on positions 4, 5, 6, and 7, were labeled as shown (see Figure 1(II)). The 4-, 5-, 6-, 7-di-substituted compounds on both $\mathrm{A}$ and $\mathrm{B}$ moieties were considered for $2 \mathrm{a}-2 \mathrm{~d}$, respectively. The 4,4'-, 5,5'-, 6,6'-, 7,7'-C-substituted compounds (3a-3d) were considered for di-substitution on the same A moiety of SSF. Furthermore, the dimeric form of $3 d$ was listed in Figure 1(III).

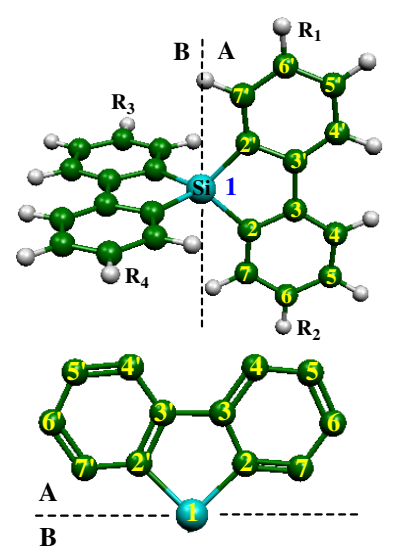

(I) $\mathrm{R}_{\mathrm{X}}$ substitution:

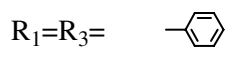

$\mathrm{R}_{2}=\mathrm{R}_{4}=\mathrm{t}-\mathrm{Bu} \quad$ la $\quad 1 \mathrm{~b}$

$\mathrm{R}_{1}=\mathrm{R}_{3}=\leadsto \mathrm{t}-\mathrm{Bu}$

$\mathrm{R}_{2}=\mathrm{R}_{4}=\mathrm{H} \quad$ le $\quad$ if

$\mathrm{R}_{1}=\mathrm{R}_{2}=\mathrm{R}_{3}=\mathrm{R}_{4}=\mathrm{H} \quad$ spirosilabifluorene $(\mathrm{SSF})$

(II) "CH"/N substitution:

$$
\begin{array}{llll}
\multicolumn{3}{c}{\mathrm{A} \text { and B }} & \mathrm{A} \\
\mathrm{X}_{4}=\mathrm{N} & 2 \mathrm{a} & \mathrm{X}_{4}=\mathrm{X}_{4}=\mathrm{N} & 3 \mathrm{a} \\
\mathrm{X}_{5}=\mathrm{N} & 2 \mathrm{~b} & \mathrm{X}_{5}=\mathrm{X}_{5^{\prime}}=\mathrm{N} & 3 \mathrm{~b} \\
\mathrm{X}_{6}=\mathrm{N} & 2 \mathrm{c} & \mathrm{X}_{6}=\mathrm{X}_{6^{\prime}}=\mathrm{N} & 3 \mathrm{c} \\
\mathrm{X}_{7}=\mathrm{N} & 2 \mathrm{~d} & \mathrm{X}_{7}=\mathrm{X}_{7^{\prime}}=\mathrm{N} & 3 \mathrm{~d}
\end{array}
$$

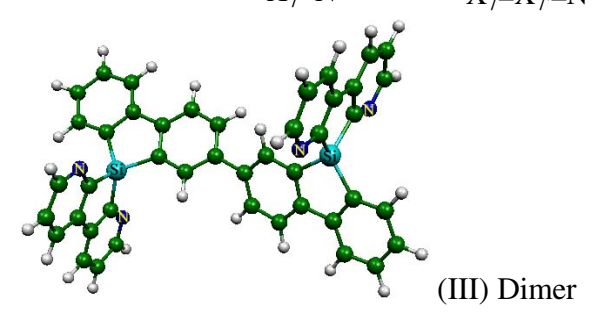

Fig. 1. Molecular structures of the investigated derivatives $(t$-Bu $=$ tert-butyl $)$ 
All the geometry optimizations for the ground state $\left(\mathrm{S}_{0}\right)$ were performed using the $a b$ initio Hartree Fock (HF) [11] and the B3LYP [12] method with the 6-31G* [13] basis set. The low-lying excited-state $\left(\mathrm{S}_{1}\right)$ structures were then optimized using the configuration interaction with single excitations (CIS) [14] with 6-31G* basis set, the optical spectra was calculated using TD-B3LYP [15] with 6-31G* basis set to obtain estimates including some account of electron correlation. All the calculations have been performed using Gaussian 03 package. [16]

\section{Results and Discussions}

\subsection{Comparison of Computational Methods and Optical Characteristics for 1a-1d}

\subsubsection{Geometries of Ground States}

More detailed insights into the nature of the excited states responsible for the optical properties can be gained by looking at the computed frontier molecular orbitals (FMOs), when calculated on a reliable molecular geometry. The optimized structures for 1a-1d show that the bond lengths and bond angles do not suffer from appreciable variation, according to different calculation methods. These derivatives keep their mutually perpendicular $\pi$-systems, and the torsion angles within silafluorene fragment are less than $1^{\circ}$. Apparently, the major differences are related to the torsion angles between substituents and the silafluorene fragments, according to HF and B3LYP results. The dihedral angles between silafluorene fragment and the phenyl/pyridyl substituents are $44^{\circ} / 26^{\circ}(1 \mathrm{a} / 1 \mathrm{~b})$ at $\mathrm{HF}$ level, and $37^{\circ} / 17^{\circ}(1 \mathrm{a} / 1 \mathrm{~b})$ at B3LYP level. This indicates that the hydrogen-free pyridine ring twists less than the phenyl group. Note that the lowest energy conformer for bipyridyl has the nitrogen atoms in a trans-conformation. The dihedral angles between the outer and inner aryl rings for biphenyl and bipyridyl substituents are $-45^{\circ}$ (1c) and $4^{\circ}(1 \mathrm{~d})$ at $\mathrm{HF}$ level, while $-37^{\circ}$ (1c) and $3^{\circ}$ (1d) at the B3LYP level. Therefore, the twisting for the backbone in $1 \mathrm{~b}$ and $1 \mathrm{~d}$ are reduced as compared with $1 \mathrm{a}$ and $1 \mathrm{c}$.

\subsubsection{Absorption Properties}

To investigate the effect of electron correlation on the computed energies and spectra, calculations using TD-B3LYP method, are carried out based on both the HF/6-31G* and B3LYP/6-31G* optimized $\mathrm{S}_{0}$ geometries. For the sake of comparison, and to estimate the accuracy of the theoretical level applied in this work, we listed both the experimental [4] and theoretical [17] maximum absorption wavelengths $\left(\lambda_{\mathrm{abs}}\right)$ for 1a-1d in Table 1. From these results, one may find that the calculated $\lambda_{\text {abs }}$ values at TD-B3LYP//HF/6-31G* level are in excellent agreement with experimental results, with less than $7 \mathrm{~nm}$ of deviation. These give credit to the computational approach, and reliable geometries may be predicted from this level, which can be applied to the system under investigation. Our results indicates that $\lambda_{\text {abs }}$ do not change significantly in 1a-1d (within the range of 314-322 nm), with only a slight blue shift being found in 1a, and while $1 \mathrm{~b}-1 \mathrm{~d}$ are nearly identical. 
Table 1. Computed absorption and emission wavelengths $\left(\lambda_{\mathrm{abs}}\right.$ and $\left.\lambda_{\mathrm{em}}, \mathrm{nm}\right)$, oscillator strength (f), assignment, coefficient

\begin{tabular}{|c|c|c|c|c|c|c|c|c|}
\hline & $\mathrm{Calc}^{\mathrm{a}}$ & $f^{\mathrm{a}}$ & Assignment & Coefficient & $\operatorname{Exp}^{\mathrm{c}}$ & $\mathrm{Calc}^{\mathrm{b}}$ & $f^{b}$ & $\mathrm{Calc}^{\mathrm{d}}$ \\
\hline \multicolumn{9}{|c|}{ Absorption } \\
\hline $1 \mathrm{a}$ & 314 & 0.200 & $\mathrm{HOMO} \rightarrow$ LUMO & 0.65 & 307 & 328 & 0.200 & 294 \\
\hline $1 b$ & 322 & 0.221 & $\mathrm{HOMO} \rightarrow$ LUMO & 0.67 & 317 & 337 & 0.180 & 309 \\
\hline $1 \mathrm{c}$ & 320 & 0.420 & $\mathrm{HOMO} \rightarrow$ LUMO & 0.64 & 315 & 338 & 0.474 & 303 \\
\hline $1 d$ & 322 & 0.267 & $\mathrm{HOMO} \rightarrow \mathrm{LUMO}$ & 0.66 & - & 337 & 0.270 & 319 \\
\hline $1 \mathrm{e}$ & 312 & 0.137 & $\mathrm{HOMO} \rightarrow$ LUMO & 0.66 & & & & \\
\hline 1f & 299 & 0.059 & $\mathrm{HOMO} \rightarrow \mathrm{LUMO}$ & 0.63 & & & & \\
\hline \multicolumn{9}{|c|}{ Emission } \\
\hline $1 \mathrm{a}$ & 385 & 0.473 & $\mathrm{HOMO} \rightarrow$ LUMO & 0.65 & 383 & & & \\
\hline $1 \mathrm{~b}$ & 387 & 0.585 & $\mathrm{HOMO} \rightarrow$ LUMO & 0.64 & 382 & & & \\
\hline $1 \mathrm{c}$ & 397 & 0.939 & $\mathrm{HOMO} \rightarrow$ LUMO & 0.67 & 393 & & & \\
\hline $1 d$ & 389 & 0.658 & $\mathrm{HOMO} \rightarrow$ LUMO & 0.69 & - & & & \\
\hline $1 \mathrm{e}$ & 382 & 0.375 & HOMO LUMO & 0.63 & & & & \\
\hline $1 \mathrm{f}$ & 364 & 0.147 & HOMO $\leftarrow$ LUMO & 0.64 & & & & \\
\hline
\end{tabular}

\subsubsection{Excited States Structures}

Comparing with the ground states structures, some of the bonds in excited states equilibrium geometries are lengthened, some others being shortened. All of the significant changes are located on one of the two identical (A or B) moieties, the other one remaining practically unaffected. The central silole ring is revealed to be the most distorted with the $\mathrm{C}_{2}-\mathrm{C}_{3}$ and $\mathrm{C}_{2},-\mathrm{C}_{3}$, bond lengths being lengthened by ca $0.06 \AA$ upon excitation. The $S_{1}$ optimized $C_{3}-C_{3}$, bond lengths in the range from 1.40 to $1.41 \AA$, are found to be shortened by ca $0.09 \AA$ compared with their corresponding $\mathrm{S}_{0}$ states. The dihedral angles between the silafluorene fragment and the phenyl/biphenyl rings decrease for $1 \mathrm{a}$ and $1 \mathrm{c}$ in the $\mathrm{S}_{1}$ states. According to the very small torsion angles $\left(\leq 1^{\circ}\right)$ between the ortho-hydrogen-free pyridine and the silafluorene fragment for $1 \mathrm{~b}$ and $1 \mathrm{~d}$ in $S_{1}$ states, effectively coplanar configurations allow better $\pi$ orbital overlap. Moreover, these molecules maintain their two mutually perpendicular $\pi$-systems in $S_{1}$ states.

\subsubsection{Emission Properties}

In Table 1, we summarize the calculation results for the first singlets excitation energies with the corresponding oscillator strengths based on TD-B3LYP//CIS/6-31G* level. The results show that, comparatively to $1 \mathrm{a}$ and $1 \mathrm{~b}, \lambda_{\mathrm{em}}$ red-shifted by $10 \mathrm{~nm}$ with an intense violet-blue emission wavelength at $397 \mathrm{~nm}$, in excellent agreement with the experimental data. The energy gaps of $1 \mathrm{a}, 1 \mathrm{~b}$, and $1 \mathrm{~d}$ do not change significantly (within the range of $3.60-3.55 \mathrm{eV})$, compared with that of $1 \mathrm{c}(3.48 \mathrm{eV})$. This is consistent with the slight red shift of $1 \mathrm{c}$, and the nearly identical wavelengths of $1 \mathrm{a}, 1 \mathrm{~b}$ and $1 \mathrm{~d}$. The oscillator strength of $1 \mathrm{c}$ is twice larger than the others. This may be due to the efficient conjugation from the external phenyl group in $S_{1}$ by decreasing the torsional angles between the external and inner phenyl rings, corresponding to some amount of orbital distribution from the external ring (see Figure 2). It also leads to a 
red-shifted wavelength for 1c. On the other hand, the pyridine and bipyridyl ring in $1 \mathrm{~b}$ and $1 \mathrm{~d}$ have no significant influence on $\lambda_{\mathrm{em}}$ compared with $1 \mathrm{a}$, which is consistent with the FMO patterns in $S_{1}$ for $1 \mathrm{a}, 1 \mathrm{~b}$, and $1 \mathrm{~d}$. FMOs are only mainly located on the silafluorene fragments and their adjacent aryl rings, the external aryl rings faily contributing to them (see Figure 2).

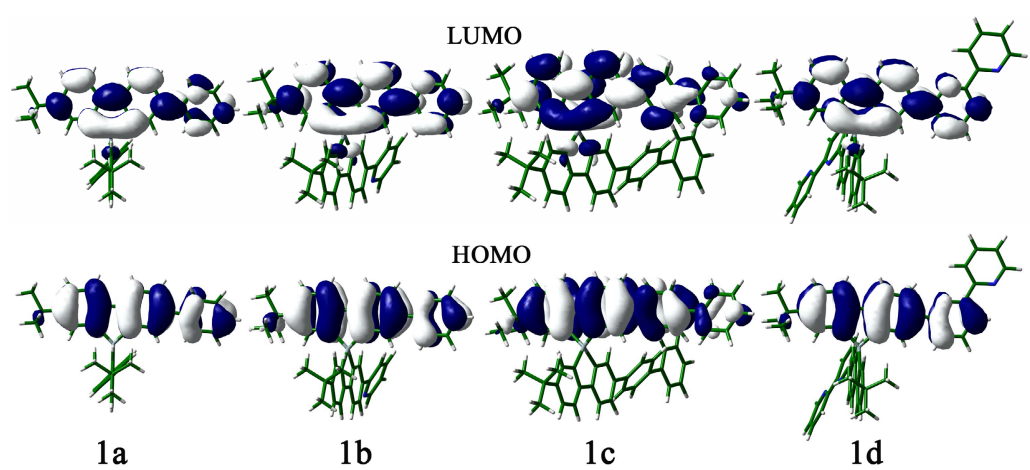

Fig. 2. TD-B3LYP/6-31G* electronic density contours of the orbitals involved in transitions for $1 \mathrm{a}-1 \mathrm{~d}$

\subsubsection{Oscillator Strength}

To investigate the role of the aryl substituent, we carried out the same calculations for $\mathrm{SSF}, 1 \mathrm{e}$, and 1f. From the results listed in Table 1, one can find that the predicted $f$ values are in the increasing order $1 \mathrm{f}<1 \mathrm{e}<1 \mathrm{a}$ for both absorption and emission results. The oscillator strength of 1e (with the bulky aromatic substituent) are close to that of $1 \mathrm{a}$ (with both the aryl and $t$-Bu substituent), compared with $t$-Bu substituted derivatives $1 \mathrm{f}$. Thus, it can be concluded that phenyl group plays a very important role in enlarging the oscillator strength.

\subsection{Optimized Geometries and Optical Properties for the "CH"/N Substituted Derivatives}

\subsubsection{Optimized Geometries of $S_{\mathbf{0}}$ and $S_{\mathbf{1}}$ States}

In general, the optimization results in $S_{0}$ do not reveal any significant change on the geometry of the skeleton of the parent molecule (SSF), when substituting "CH" by N. In all the cases, the silafluorene fragments maintain their mutually perpendicular $\pi$-systems. Compared with the skeleton of SSF, the bond lengths and bond angles change mainly on the nitrogen and its neighboring carbon atoms. The $\mathrm{C}-\mathrm{N}$ bond lengths are predicted to be ca. $1.321 \AA$, the C-C-N and N-C-C angles being larger (by ca 1.32 $5.40^{\circ}$ ) than the corresponding $\mathrm{C}-\mathrm{C}-\mathrm{C}$ angle in SSF. However, the $\mathrm{C}-\mathrm{N}-\mathrm{C}$ bond angles become smaller (by ca $2.76-4.48^{\circ}$ ) than the corresponding C-C-C ones in SSF. The optimization results for $S_{1}$ states possess the same feature as 1a-1d, upon $S_{0}-S_{1}$ transition, the main is localized on one of the two identical (A or B) moieties, the other parts remaining practically unaffected. 


\subsubsection{Emission Properties}

Figure 3 shows the electronic density contours of the FMOs for SSF, 2a, and 3a in $S_{1}$ states as representatives of systems under investigation. Both the HOMO and LUMO spread over $\pi$-conjugated backbone as those for 1a-1d. They are respectively antibonding and bonding between the carbon atoms bridging the two phenyl groups within the silafluorene fragment for HOMOs and LUMOs. The same bonding-antibonding pattern is also observed between adjacent atoms in the same aryl ring.

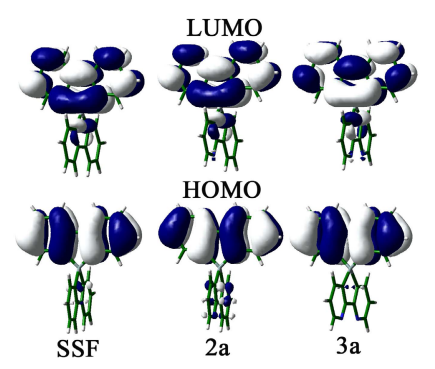

Fig. 3. TD-B3LYP/6-31G* electronic density contours of the LUMO (upper panel) and HOMO (lower panel) for spirosilabifluorene, $2 \mathrm{a}$ and $3 \mathrm{a}$

The calculated emission wavelengths $\left(\lambda_{\mathrm{em}}\right)$, the oscillator strength $(f)$, the transition, corresponding assignment, and the main $\mathrm{CI}$ expansion of the derivatives of interest are listed in Table 2. For all "CH"/N substituted derivatives, emitting states are mainly of LUMO $\rightarrow$ HOMO character. As there is a slight change for the energy gaps $(E g)$ between HOMO and LUMO for the investigated system, slight change can be also found for $\lambda_{\mathrm{em}}$, with the maximum change being $13 \mathrm{~nm}$ (in $2 \mathrm{c}$ and $3 \mathrm{~d}$ ).

Table 2. Computed emission wavelengths $\left(\lambda_{\text {em }}\right)$, oscillator strength $(f)$, transitions, coefficient

\begin{tabular}{|c|c|c|c|c|c|}
\hline & $\lambda_{\mathrm{em}}(\mathrm{nm})$ & $f$ & transition & Assignment & coefficient \\
\hline $2 a$ & 357 & 0.093 & $\mathrm{~S}_{1} \rightarrow \mathrm{S}_{0}$ & $\mathrm{HOMO} \leftarrow \mathrm{LUMC}$ & 0.64 \\
\hline $2 b$ & 367 & 0.097 & $\mathrm{~S}_{1} \rightarrow \mathrm{S}_{0}$ & $\mathrm{HOMO} \leftarrow \mathrm{LUMC}$ & 0.64 \\
\hline $2 \mathrm{c}$ & 347 & 0.090 & $\mathrm{~S}_{1} \rightarrow \mathrm{S}_{0}$ & $\mathrm{HOMO} \leftarrow \mathrm{LUMC}$ & 0.64 \\
\hline $2 d$ & 361 & 0.070 & $\mathrm{~S}_{1} \rightarrow \mathrm{S}_{0}$ & $\mathrm{HOMO} \leftarrow \mathrm{LUMC}$ & 0.63 \\
\hline $3 a$ & 362 & 0.100 & $\mathrm{~S}_{1} \rightarrow \mathrm{S}_{0}$ & $\mathrm{HOMO} \leftarrow \mathrm{LUMC}$ & 0.64 \\
\hline $3 b$ & 363 & 0.100 & $\mathrm{~S}_{1} \rightarrow \mathrm{S}_{0}$ & $\mathrm{HOMO} \leftarrow \mathrm{LUMC}$ & 0.64 \\
\hline $3 c$ & 363 & 0.100 & $\mathrm{~S}_{2} \rightarrow \mathrm{S}_{0}$ & $\mathrm{HOMO} \leftarrow \mathrm{LUMC}$ & 0.64 \\
\hline $3 d$ & 373 & 0.012 & $\mathrm{~S}_{2} \rightarrow \mathrm{S}_{0}$ & $\mathrm{HOMO} \leftarrow \mathrm{LUMC}$ & 0.68 \\
\hline
\end{tabular}

\subsection{Optical Property for Dimer}

It is believed that larger $f$ can also be reached in the case of oligomers. Moreover, both absorption and emission wavelengths will be red-shifted in the case of oligomers. [18] In this work, we considered the dimeric form of $3 \mathrm{~d}$ through positions 6 and 6' (shown in Figure 1(III)). On the basis of the same approach, the $\lambda_{\text {abs }}$ values for the first three states are predicted to be $346 \mathrm{~nm}(f=0.0000), 346 \mathrm{~nm}(f=0.0072)$ and $329 \mathrm{~nm}(f=0.6828)$, respectively, in comparison with $3 \mathrm{~d}$, whose $\lambda_{\text {abs }}$ corresponding to the first three sates are 
$328 \mathrm{~nm}(f=0.000), 323 \mathrm{~nm}(f=0.000)$ and $307 \mathrm{~nm}(f=0.000)$, it becomes clear that both $\lambda_{\text {abs }}$ and $f$ are increased in the dimeric form. The $\lambda_{\text {em }}$ values for the first three excited states are predicted to be $405 \mathrm{~nm}(f=1.184), 395 \mathrm{~nm}(f=0.000)$ and $395 \mathrm{~nm}$ $(f=0.008)$, respectively, while those of $3 \mathrm{~d}$ are $380 \mathrm{~nm}(f=0.000), 373 \mathrm{~nm}(f=0.012)$ and $353 \mathrm{~nm}(f=0.042)$. It can be found that both $\lambda_{\mathrm{abs}} / \lambda_{\mathrm{em}}$ and $f$ are increased in the dimmer form. In general, larger oscillator strengths correspond to the intensive fluorescent emitting, therefore, this approach may contribute to develop the property of emitting materials.

\section{Conclusions}

Calculations have been performed on a novel class of spirosilabifluorene and some of its derivatives. Some light is shed on a series of experimentally available derivatives and " $\mathrm{CH}$ "/N on substituted derivatives. Our calculation results on 1a-1d are in good agreement with experimental results. The similar optical properties for $1 \mathrm{a}-1 \mathrm{~d}$ are ascribed to the identical FMO distribution pattern and similar energy gaps between FMOs. Compared to the pristine molecule, no significant change of emission wavelengths is found for the "CH"/N substituted derivatives. According to our results, phenyl group and oligomers may play a very important role in enlarging the oscillator strengths, which will increase the fluorescent intensity. Thus, we may be improved the emitting properties by adding the aryl substituents or oligomerization. Furthermore, the dimeric forms of the designed " $\mathrm{CH}$ "/ $\mathrm{N}$ substituted derivatives are expected to be promising candidate for blue emitting materials.

Acknowledgment. Financial supports from the NSFC (No.50473032) and Outstanding Youth Project of Jilin Province are gratefully acknowledged.

\section{References}

1. Tang, C. W., Van Slyke, S. A.: Organic electroluminescent diodes. Appl. Phys. Lett. 51(1987) 913-915.

2. Kulkarni, A. P., Tonzola, C. J., Babel, A., Jenekhe, S. A. Electron Transport Materials for Organic Light-Emitting Diodes. Chem. Mater. 16 (2004) 4556-4573.

3. Tu. G., Zhou, Q., Cheng, Y., Wang, L.X.: White electroluminescence from polyfluorene Chemically doped with 1,8-napthalimide moieties. Appl. Phys. Lett. 85(2004) 2172-2174.

4. Lee, S. H., Jang, B. B., Kafafi, H. Z.: Highly Fluorescent Soil-State Asymmetric Spirosilabifluorene Derivatives. J. Am. Chem. Soc. 127(2005) 9071-9078.

5. Ohshita, J., Lee, K. H., Hamamoto, D., Kunugi, Y., Ikadai, J., Kwak, Y. W.: Synthesis of Novel Spiro-condensed Dithienosiloles and the Application to Organic FET. Chem. Lett. 33(2004) 892-893.

6. Schneider, D., Rade, T., Riedl, T., Dobbertin, T., Werner, O.: Deep blue widely tunable organic solid-state laser based on a spirobifluorene derivative. Appl. Phys. Lett. 84(2004), 4693-4695.

7. Yin, S. W., Yi, Y.P., Li, Q. X., Yu, G., Liu, Y. Q., Shuai, Z. G.: Balanced Carrier Transports of Electrons and Holes in Silole-Based Compounds-A Theoretical Study. J. Phys. Chem. A. 110(2006) 7138-7143. 
8. Lukeš, V., Pálszegir, T., Milota, F., Sperling, J., Kauffmann, H. F.: Density Matrix Analysis, Simulation, and Measurements of Electronic Absorption and Fluorescence Spectra of Spirobifluorenes. J. Phys. Chem. A. 110(2006) 1775-1782.

9. Gahungu, G., Zhang, J. P.: "CH”/N substituted mer-Gaq3 and mer-Alq3 derivatives; an effective approach for the tuning of emitting color. J. Phys. Chem. B. 109 (2005) 17762-17767.

10. Van Slyke, S. A., Bryan, P. S., Lovencchio, F. V.; US Patent 1990, 5, 150, 006.

11. McWeeny, R. Dierksen. G.: Self-Consistent Perturbation Theroy. II. Exctension to Open Shells. J. Chem. Phys. 49(1968) 4852-4856.

12. Becke; A. B.: Density-functional thermochemistry. III. The role of exact Exchange. J. Chem. Phys. 98(1993) 5648-5652.

13. Binkley, J. S., Pople J. A., Hehre, W. J.:Self-Consistent Molecular Orbital Theory for Excited States. J. Am. Chem. Soc. 102 (1980) 939-947.

14. Foresman, J. B., Head-Gordon, M., Pople, J. A., Frisch, M. J.: Toward a Systematic Molecular Orbital Theory for Excited States. J. Phys. Chem. 96 (1992) 135-149.

15. Stratman, R. E., Scuseria, G. E., Frisch, M. J.: An efficient implementation of time-dependent density-functional theory for the calculation of excitation energies of large molecules. J. Chem. Phys. 109(1998) 8218-8224.

16. Frisch, M. J., et al. Gaussian, Inc., Pittsburgh PA, 2003. Gaussian 03, Revision B.03.

17. Yang, G. C., Su, Z. M., Qin, C. S.: Theoretical Study on the Second-Order Nonlinear Optical Properties of Asymmetric Spirosilabifluorene Derivatives. J. Phys. Chem. A. 110 (2006) 4817-4821.

18. Yang, L., Feng, J. K., Ren, A. M.: Theoretical Study on Electronic Structure and Optical Properties of Phenothiazine-Containing Conjugated Oligomers and Polymers. J. Org. Chem. 70 (2005) 5987-5996. 